\title{
Development and application of a Japanese model of the WHO fracture risk assessment tool (FRAX ${ }^{\mathrm{TM}}$ )
}

\author{
S. Fujiwara $\cdot$ T. Nakamura $\cdot$ H. Orimo $\cdot$ T. Hosoi $\cdot$ \\ I. Gorai • A. Oden • H. Johansson • J. A. Kanis
}

Received: 5 November 2007 / Accepted: 10 December 2007 / Published online: 22 February 2008

(C) International Osteoporosis Foundation and National Osteoporosis Foundation 2007

\begin{abstract}
Summary The present study estimated the 10-year probability using the Japanese version of WHO fracture risk assessment tool $\left(\mathrm{FRAX}^{\mathrm{TM}}\right)$ in order to determine fracture probabilities that correspond to intervention thresholds currently used in Japan and to resolve some issues for its use in Japan.
\end{abstract}

\footnotetext{
S. Fujiwara $(\bowtie)$

Department of Clinical Studies,

Radiation Effects Research Foundation,

Hiroshima, Japan

e-mail: fujiwara@rerf.or.jp

T. Nakamura

Department of Orthopedic Surgery,

University of Occupational and Environmental Health,

Kitakyushu, Japan

H. Orimo

Japan Osteoporosis Foundation,

Tokyo, Japan

T. Hosoi

Department of Advanced Medicine,

National Center for Geriatrics and Gerontology,

Obu, Japan

I. Gorai

Department of Obstetrics \& Gerontology,

International University of Health \& Welfare, Atami Hospital,

Atami, Japan

\section{A. Oden $\cdot$ H. Johansson $\cdot$ J. A. Kanis}

WHO Collaborating Centre for Metabolic Bone Diseases,

University of Sheffield Medical School,

Sheffield, UK

e-mail: w.j.pontefract@sheffield.ac.uk
}

Introduction The objective of the present study was to evaluate a Japanese version of the WHO fracture risk assessment $\left(\mathrm{FRAX}^{\mathrm{TM}}\right)$ tool to compute 10-year probabilities of osteoporotic fracture in Japanese men and women. Since lumbar spine bone mineral density (BMD) is used preferentially as a site for assessment, and densitometers use Japanese reference data, a second aim was to investigate the suitability and impact of this practice in Japan.

Methods Fracture probabilities were computed from published data on the fracture and death hazards in Japan. Probabilities took account of age, sex, the presence of clinical risk factors and femoral neck BMD. Fracture probabilities were determined that were equivalent to intervention thresholds currently used in Japan. The difference between $\mathrm{T}$-scores derived from international reference data and that using Japanese-specific normal ranges was estimated from published sources. The gradient of risk of BMD for fracture in Japan was compared to that for BMD at the lumbar spine in the Hiroshima cohort.

Results The 10-year probabilities of a major osteoporosisrelated fracture that corresponded to current intervention thresholds ranged from approximately $5 \%$ at the age of 50 years to more than $20 \%$ at the age of 80 years. The use of femoral neck BMD predicts fracture as well as or better than BMD tests at the lumbar spine. There were small differences in T-scores between those used for the model and those derived from a Japanese reference population.

Conclusions The FRAX ${ }^{\mathrm{TM}}$ tool has been used to determine possible thresholds for therapeutic intervention, based on equivalence of risk with current guidelines. The approach will need to be supported by appropriate health economic analyses. Femoral neck BMD is suitable for the prediction of fracture risk among Japanese. However, when applying the FRAX $^{\mathrm{TM}}$ model to Japan, T-scores and Z-scores should be converted to those derived from the international reference. 
Keywords Bone mineral density · Fracture · Fracture probability . Fracture risk assessment tool $\cdot$ Intervention thresholds · Japan

\section{Introduction}

Fractures related to osteoporosis have become a major health and economic burden in Asian countries just as they have in North America and Europe. An estimated 117,900 cases of hip fracture occurred in 2002 [1], and the incidence in Japan has increased in the past 10 years [1,2]. Asia will be expected to have the highest absolute increase in fracture number because it has the largest population. Early detection of individuals with high fracture risk using clinical risk factors would have a substantial impact on reducing the burden of fractures in Asia.

A series of meta-analyses on prospective populationbased cohorts has identified a number of clinical risk factors that contribute to fracture risk independently of BMD at the femoral neck [3]. The integration of these risks would, therefore, enhance the predictive value of BMD [4]. The risk factors comprise age, sex, bone mineral density, body mass index (BMI), long-term use of glucocorticoids, parental history of hip fracture, history of fragility fracture, smoking, alcohol consumption (3 or more units/day), and secondary osteoporosis such as rheumatoid arthritis. A WHO scientific group has proposed that the 10-year probability for fracture is used to express fracture risk for clinical assessment [5] and to determine intervention thresholds [3] The aim of this study was to create a fracture probability model based on the methodology of the WHO risk assessment tool $\left(\mathrm{FRAX}^{\mathrm{TM}}\right)$ [6] calibrated to the epidemiology of Japan.

In addition, several problems need to be resolved before the FRAX ${ }^{\mathrm{TM}}$ model is applied to Japan. First, the FRAX ${ }^{\mathrm{TM}}$ tool inputs femoral neck BMD and the Z-score or T-score is based on the NHANES III reference data base. In Japan, BMD at the lumbar spine is widely used clinically because the physical size of Japanese people is smaller than that of Western people, giving rise to a view based on little evidence that the reproducibility of measurements at the femoral neck BMD would be poorer than that at the lumbar spine. Furthermore, data on the young adult mean (YAM) and the mean at each age are installed in the DXA systems in Japan, and programmed to calculate T- and Z-scores from Japanese reference data. In addition, the Japanese Society for Bone and Mineral Research [7, 8] provide recommendations for the diagnosis of osteoporosis and intervention based on YAM, and these are widely used in clinical practice.

Against this background, additional aims of the present study were to provide fracture probabilities based on the
FRAX $^{\text {TM }}$ tool that were equivalent to currently accepted intervention thresholds, explore the impact of using Japanese-specific normative data for femoral neck BMD, and reassess the respective performance characteristics of BMD at the femoral neck and lumbar spine.

\section{Methods}

Models were constructed to compute the 10-year probability of hip fracture and a major osteoporosis-related fracture in Japan. A major osteoporosis-related fracture was defined as a clinical spine, hip, proximal humeral and forearm fracture. Poisson modelling was used to calculate the hazard functions. The relationship between probability and hazard functions were used to calculate the 10-year probability or fracture for a combination of the risk factors. The mortality estimates for Japan were those published by the World Health Organization for 1999, which accord with estimates from Japan [9]. The incidence of hip fractures was taken from previously published sources [1] as was the incidence of fractures at the proximal humerus and distal forearm [10]. Since the incidence of a clinical vertebral fracture was not known in Japan, we assumed that the ratio of clinical vertebral fracture incidence to that of a vertebral fracture diagnosed by radiographic surveys [11] would be the same in the Japan as it was for Sweden [12].

The relationship of clinical risk factors to fracture outcomes was assumed to be the same as that determined in a large meta-analysis of risk factors of 190,000 patient years from nine prospectively studied population-based cohorts from Europe, Australia, North America and Asia [3]. The relationship has been validated in a further 11 cohorts of population-based samples with 1.2 million patient years of observation from the same regions [4]. The independent contribution of each risk factor was used to compute probabilities of fracture in the absence of clinical risk factors or in the presence of any combination $[13,14]$.

In Japan, the criteria for the diagnosis of osteoporosis prepared by the Japanese Society for Bone and Mineral Research [7] are based on BMD measurements expressed as a percentages of the young adult mean (YAM) for women. In patients with no prior fragility fracture a diagnosis of osteoporosis is made where the BMD is less than $70 \%$ of YAM. In patients with a previous fracture, osteoporosis is diagnosed where the BMD is less than $80 \%$ of YAM. These diagnostic thresholds, derived by maximising sensitivity and specificity for fracture detection, are also used as intervention thresholds. In order to compare intervention thresholds using YAM with probabilities derived from the FRAX ${ }^{\mathrm{TM}}$ algorithm, T-score equivalents were used. The T-score equivalent to $70 \%$ and $80 \%$ of 
YAM for Japanese people is $-2.7 \mathrm{SD}$ and $-1.8 \mathrm{SD}$, respectively, using the NHANES III reference for BMD at the femoral neck in Caucasian women aged 20-29 years [15].

The relative performance characteristics of BMD at the lumbar spine and femoral neck were examined in a population-based prospective study in Hiroshima. The Hiroshima cohort comprised 2,596 men and women (69\% female, 9,803 person years, mean age 65.1 years). Details of the cohort have been previously published [11]. In brief, the participants received measurement of lumbar spine and femoral neck BMD using dual X-ray absorptiometry (DXA, QDR-2000, Hologic) during the period from 1994 to 1995 and were followed for a mean period of 4 years. Information about hip fracture, fracture of the distal radius, proximal humeral fracture and clinical spinal fracture was collected at interview by trained nurses and physicians during the biennial health examinations. One hundred eighty-six fractures were detected during the follow-up period, of which 89 were categorized as osteoporotic fractures and 31 were hip fractures. The gradient of fracture risk (increase in fracture risk per SD change in Z-score for BMD and increase in fracture risk per $0.1 \mathrm{~g} / \mathrm{cm}^{2}$ change in BMD) at the two sites was determined by the use of Poisson models [16]. The fracture outcomes were calculated for hip fracture, a major osteoporosis-related fracture (femoral neck, distal radius, proximal humerus and clinical spine fracture) and all fractures.

The mean height and body weight for Japanese women in the Hiroshima cohort was $150 \mathrm{~cm}$ and $52.6 \mathrm{~kg}$, respectively, giving a BMI of $23.4 \mathrm{~kg} / \mathrm{m}^{2}$. The calculation of fracture probability was made at this BMI, but differences in BMI have little effect on predictive value for fracture risk assessment in the presence of BMD [17].

Japanese-specific T- and Z-scores and those derived from NHANES III were compared using the database of the Japanese Society for Bone and Mineral Research [7]. Data using both methods of calculation were entered into the FRAX $^{\text {TM }}$ tool.

\section{Results}

Ten-year probability of fracture

The 10-year probability of a major osteoporotic fracture for individuals without clinical risk factors is shown in Fig. 1 grouped by sex, age and T-score. The 10-year probability increased with age and with decreasing T-score. At younger ages, the fracture probability was similar in men and women. With advancing age, probabilities rose as expected, but the increase was greater in women than in men. In men aged 80 years, the 10-year probability for osteoporosis- related fracture exceeded $10 \%$ at a T-score of $-3 \mathrm{SD}$, whereas in women of the same age, fracture probabilities exceeded $10 \%$ with T-score of -1 SD.

The contribution of clinical risk factors to fracture probability is shown in Fig. 2 for women aged 65 years with a BMI of $23.4 \mathrm{~kg} / \mathrm{m}^{2}$. In women without clinical risk factors, the 10-year probability for an osteoporosis-related fracture was $7.5 \%$. The 10 -year probability was higher in the presence of clinical risk factors. Smoking and alcohol were relatively weak risk factors, the use of long-term glucocorticoids of intermediate weight, and a parental history of hip fracture or a prior fragility fracture were associated with the highest risks. For example, the 10-year probability was $8.1 \%$ for smokers and $14.5 \%$ for individuals with a prior fracture. The 10-year probability for hip fracture was $1.1 \%$ in women without a clinical risk factor, $1.6 \%$ in smokers and $2.7 \%$ in women with a previous fracture (see Fig. 2).

Fracture probabilities were computed in women at the diagnostic threshold recommended in Japan. Thus, the cutoff level of BMD was set at $70 \%$ of YAM in women without a previous fracture and at $80 \%$ of YAM in those with a previous fragility fracture. In women aged 50, 60, 70 and 80 years without clinical risk factors and with BMD equivalent to $70 \%$ of YAM, the 10 -year probability was $5.4 \%, 8.7 \%, 13.8 \%$ and $23 \%$, respectively. In women having BMD equivalent to $80 \%$ of YAM and existing fracture but no other clinical risk factors, the 10-year probability was $7.1 \%, 10.5 \%, 14.7 \%$ and $23.4 \%$ at the same ages, respectively. Thus, at each age, the fracture probability was similar using the two diagnostic criteria. In contrast, the fracture probability equivalent to the diagnostic threshold in Japan rose with age, and at the age of 80 years was about four times higher than that at age 50 years (Fig. 3). Similar findings were apparent for hip fracture probability in that probabilities equivalent to the diagnostic threshold in Japan rose with age. The increase with age was more marked than for all major fractures and at the age of 80 years was about 6-40 times higher than that at age 50 years depending on the threshold used (see Fig. 3).

\section{Comparison of lumbar spine and femoral neck BMD}

The gradient of fracture risk for spine BMD and femoral neck BMD in the Hiroshima cohort indicated that lumbar spine measurements predicted all fractures, osteoporosisrelated fracture and hip fracture with approximately equal gradients of risk that ranged from $1.25 / \mathrm{SD}$ for all fractures to $1.17 / \mathrm{SD}$ for hip fractures. There was no difference in the gradient of risk between men and women. In the case of hip fracture risk, the gradient of risk in men and women combined was not statistically significant with BMD measured at the lumbar spine. BMD at femoral neck had 
Fig. 1 Ten-year probability (\%) of osteoporotic fracture (hip, clinical spine, humerus, forearm) in Japanese men and women without clinical risk factors according to age and Tscore for BMD at the femoral neck
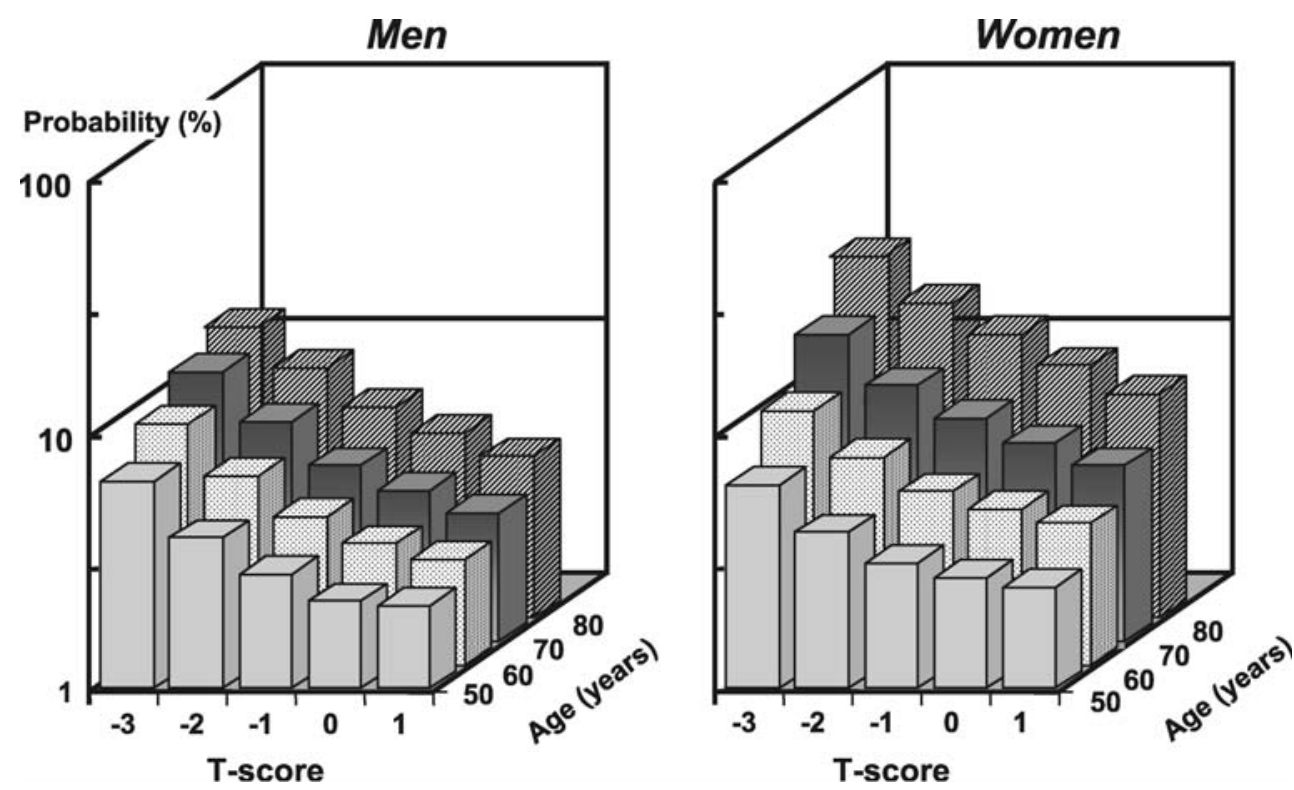

a similar or slightly higher gradient of risk for fractures compared with spine BMD, particularly in the case of hip fracture (Table 1). There was no significant difference in gradient of risk between lumbar spine BMD and femoral neck BMD with the exception of that for hip fracture where the gradient of risk was significantly higher for measurements made at the femoral neck. When gradient of risk was standardized to a constant denominator (i.e., RR/0.1 g/ $\mathrm{cm}^{2}$ ) the findings remained unchanged (see Table 1).

Japanese reference values

The reference mean in women aged 20-29 years at the femoral neck was $0.858 \mathrm{~g} / \mathrm{cm}^{2}\left(\mathrm{SD}=0.120 \mathrm{~g} / \mathrm{cm}^{2}\right)$ using the NHANES III data. When young normal values were computed from the Japanese population the mean BMD was $0.786 \mathrm{~g} / \mathrm{cm}^{2}\left(\mathrm{SD}=0.107 \mathrm{~g} / \mathrm{cm}^{2}\right)$. Thus the threshold for osteoporosis using the NHANES III data was $0.558 \mathrm{~g} / \mathrm{cm}^{2}$ and that derived from the Japanese data was $0.519 \mathrm{~g} / \mathrm{cm}^{2}$. The thresholds for osteopenia (WHO definition) were $0.738 \mathrm{~g} / \mathrm{cm}^{2}$ and $0.679 \mathrm{~g} / \mathrm{cm}^{2}$, respectively. Thus there were systematic differences in the T-score derived from the two data sets. A comparison of fracture probabilities computed from the z-scores using the two approaches is shown in Fig. 4 for different combinations of risk factors. The differences in probabilities were relatively modest, but as expected, the use of Japanese reference values overestimated fracture probabilities.

\section{Discussion}

This paper describes the development of the WHO fracture risk assessment tool calibrated to the epidemiology of
Fig. 2 Ten-year probability for osteoporotic (hip, clinical spine, humerus, forearm) and hip fracture $(\%)$ according to the presence of a clinical risk factor, in women at the age of 65 years and with a BMI of $23.4 \mathrm{~kg} / \mathrm{m}^{2}$
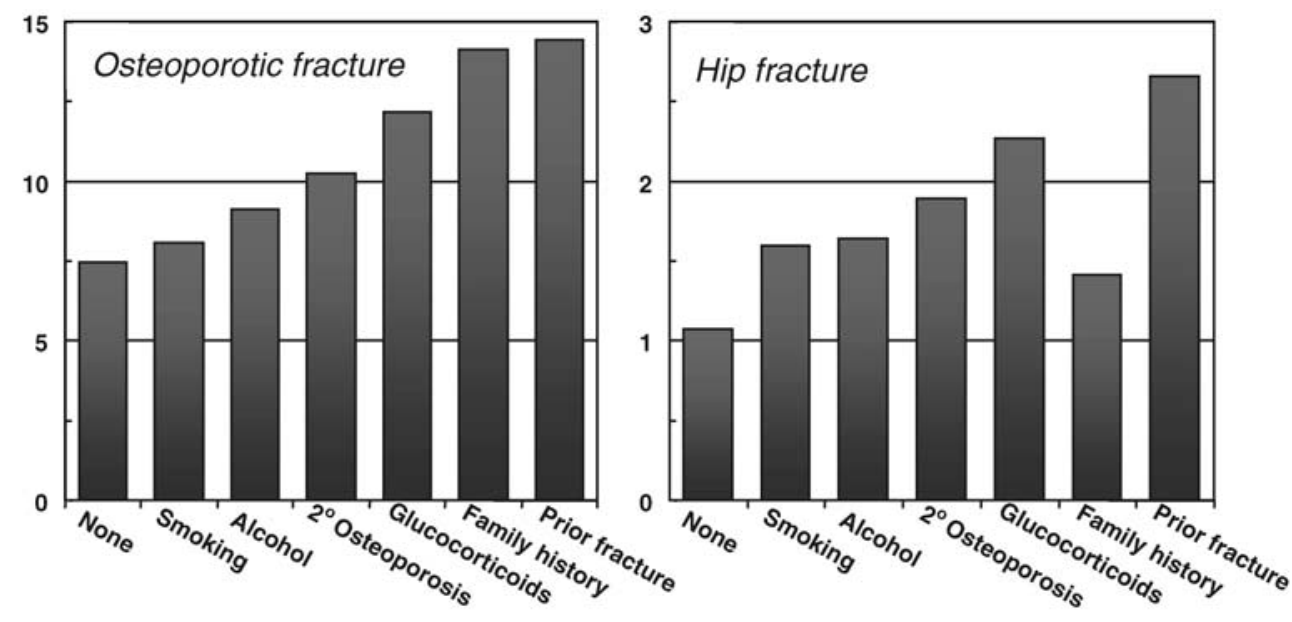
Fig. 3 Ten-year probability of osteoporotic (hip, clinical spine, humerus, forearm) and hip fracture based on women at the threshold for the diagnosis of osteoporosis using the criteria of the Japanese Bone Mineral Metabolism Association 10-year fracture* probability (\%)

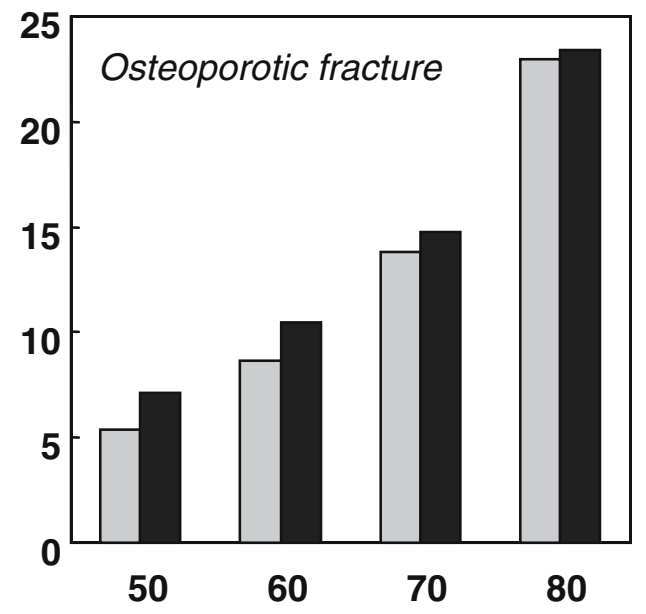

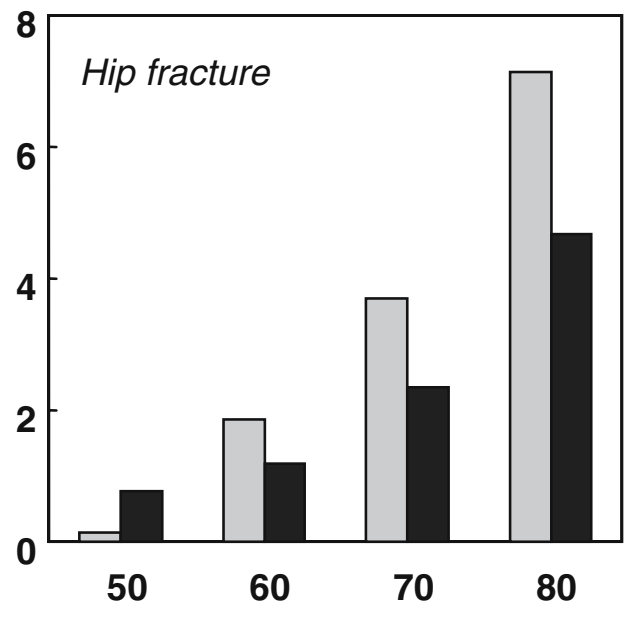

Age (years)
Japan. The incidence of hip fracture, distal radius fracture and proximal humeral fracture in Japan is lower than that in North America or Northern Europe [1, 10]. However, the prevalence and incidence of spinal fracture are higher in Japan $[11,18]$. A minority of all cases of morphological spinal fracture are assumed to be clinical spine fractures in the Japanese FRAX ${ }^{\mathrm{TM}}$ models. The multiplier is age and sex specific. For men the multiplier goes from approximately $33 \%$ at age 50 to $48 \%$ at age 85 . For women the corresponding figures are $19 \%$ and $24 \%$. These estimates, derived from the epidemiology of fracture in Sweden [12], have been shown to hold true for Japan [19].

The FRAX ${ }^{\mathrm{TM}}$ algorithm is suitable for assessment in men and women from the age of 40 years and calculates the 10 -year probability for both hip fracture and a major

Table 1 Predictive ability of spine and femoral neck BMD for any, osteoporotic and hip fracture in men and women from Japan

\begin{tabular}{llllll}
\hline & \multicolumn{2}{l}{$\mathrm{RR} / \mathrm{SD}$} & & \multicolumn{2}{l}{$\mathrm{RR} / 0.1 \mathrm{~g} / \mathrm{cm}^{2}$} \\
\cline { 6 - 6 } \cline { 5 - 6 } & $\mathrm{RR}$ & $95 \% \mathrm{CI}$ & & $\mathrm{RR}$ & $95 \% \mathrm{CI}$ \\
\hline $\begin{array}{l}\text { a. Any fracture } \\
\text { Femoral neck }\end{array}$ & 1.45 & $1.23-1.70$ & & 1.43 & $1.22-1.68$ \\
$\begin{array}{l}\text { Lumbar spine } \\
\text { b. Osteoporosis-related fractures }\end{array}$ & 1.25 & $1.13-1.39$ & & 1.38 & $1.19-1.61$ \\
$\begin{array}{l}\text { Femoral neck } \\
\text { Lumbar spine }\end{array}$ & 1.40 & $1.09-1.78$ & & 1.38 & $1.09-1.74$ \\
c. Hip fracture & 1.20 & $1.04-1.40$ & & 1.30 & $1.05-1.61$ \\
Femoral neck & $2.08^{\mathrm{b}}$ & $1.34-3.22$ & & 2.11 & $1.38-3.23$ \\
Lumbar spine & 1.17 & $0.91-1.50$ & & 1.25 & $0.87-1.80$ \\
\hline
\end{tabular}

${ }^{\text {a }}$ Hip, clinical spine, forearm and proximal humerus

b Significantly higher than lumbar spine $(\mathrm{P}=0.049)$ osteoporosis-related fracture. One of its strengths is that it can capture the independent contribution of several clinical risk factors to fracture risk and can be used with or without information on femoral neck BMD. A more detailed account of the properties of the FRAX ${ }^{\mathrm{TM}}$ models is provided elsewhere [13]. In this paper, we focused on its application to decision-making in clinical practice with the estimation of intervention threshold i.e., the fracture probability at which intervention is currently considered to be worthwhile. The approach should be distinguished from intervention thresholds that are based on health economic analysis.

The WHO makes no specific recommendation concerning intervention thresholds, since these depend on many local factors [6]. Rather, it is suggested they should be determined by each country, based on the local healthcare situation and cost-effectiveness of the treatment of osteoporosis. Intervention thresholds, based on cost-effectiveness have been formulated in the UK, the USA and in Sweden [20-22]. In Japan, diagnostic thresholds are used as intervention thresholds. When the probabilities of osteoporosis-related fracture were determined at these thresholds, they varied with age (see Fig. 3), ranging from approximately $5 \%$ at the age of 50 years to more than $20 \%$ at the age of 80 years. Against this background, a 10-year probability of $10 \%$ for osteoporosis-related fracture may be an acceptable intervention threshold for Japan, though an optimization should take account of health economic consequences for individuals and for the health care budget.

The FRAX ${ }^{\mathrm{TM}}$ tools are designed to be extensively used in the world as a means of identifying individuals with elevated risk for fracture and aid in the determination of the threshold for therapeutic intervention, but there will be hurdles to be faced in the ease of its acceptance. Such 
Fig. 4 Correlation between 10 year fracture probabilities (\%) in women without clinical risk factors computed from normative data using NHANES III reference values and Japanesederived reference values for femoral neck BMD. BMI is set at $23.4 \mathrm{~kg} / \mathrm{m}^{2}$

\section{Ten-year probability (\%) Japan}

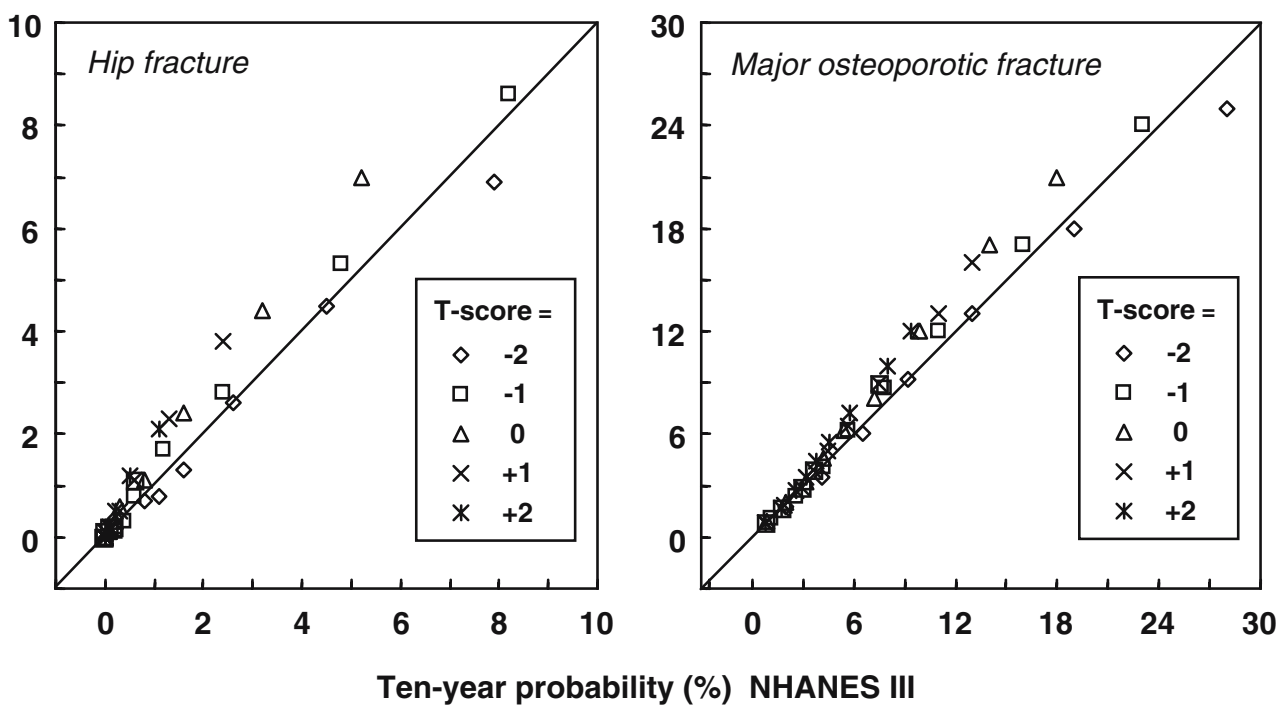

hurdles are likely to differ from country to country. In Japan, the choice of clinical risk factors is not at issue since the risk factors adopted in the FRAX ${ }^{\mathrm{TM}}$ algorithms included the data from the Hiroshima cohort, Japan [11, 23, 24], and the validation included the Japanese Miyama cohort [13]. More problematic is the inclusion of femoral neck BMD, since the lumbar spine measurement is the most widely used in Japan. Asian physiques are smaller than those of Caucasians, and the geometric characteristics of the femoral neck in Japanese differ from those in American women, in that the femoral neck length is shorter than in the Japanese $[25,26]$. Because of uncertainty regarding measurement of femoral neck BMD, measurement of lumbar BMD is widely used in Japan. The present study indicates that concerns over the use of femoral neck BMD are unfounded.

Femoral neck BMD was superior in its ability to predict hip fractures compared with spine BMD, and BMD at the spine and femoral neck had similar predictive value for fractures other than hip fracture. Fujiwara et al. [11] have also shown that the ability to predict the risk for morphological spinal fracture was similar between femoral neck BMD and lumbar spine BMD. These data on gradients of fracture risk, derived in Japan, did not differ from those reported in Western countries. Indeed, the meta-analysis used to inform the FRAX ${ }^{\mathrm{TM}}$ tool showed no evidence for heterogeneity in gradients of risk between cohorts [16]. Thus the evidence suggests that fracture risk assessment is not disadvantaged by the use of femoral neck BMD. Indeed, the converse may be true.

A further hurdle, unique to Japan, is that diagnostic thresholds differ from the WHO description of osteoporosis which defines osteoporosis on the basis of a fixed T-score threshold $(\leq-2.5 \mathrm{SD})$ using an international reference standard for young (aged 20-29 years) Caucasian women [15]. In Japan, diagnostic thresholds are also derived by reference to a young population, but differ from the WHO in that a local (i.e., Japanese) standard is used and that the criteria differ in patients with or without previous fracture. In view of the widespread use of data derived from the Japanese population, the question arises whether T-scores or Z-scores derived from Japanese databases could be used in the FRAX ${ }^{\mathrm{TM}}$ tool, rather than those derived from the international reference base. In the present study, mean BMD at the femoral neck was lower in Japanese women than in the NHANES III sample from the USA, as previously shown [27]. The difference was not, however, large (approximately a half a SD). There were also differences in the SD which was smaller in Japanese women than in the NHANES III sample $(0.107$ and $0.120 \mathrm{~g} /$ $\mathrm{cm} 2$, respectively). Not surprisingly, the DXA-based T-score obtained from Japanese and USA populations differed as did the computed probabilities. Although the differences were small at low T-scores, when applying the FRAX ${ }^{\mathrm{TM}}$ model to Japan, it is preferable to program the system so that the Japanese T- and Z-scores are converted into the appropriate Tand Z-scores based on NHANES III.

In conclusion, a FRAX ${ }^{\mathrm{TM}}$ tool has been developed to compute fracture probabilities calibrated to the epidemiology of Japan. The tool has been used to determine possible thresholds for therapeutic intervention, based on equivalence of risk with current guidelines. The approach will need to be supported by appropriate health economic analyses. The present study indicates that the femoral neck BMD is suitable for prediction of the risk for fracture among Japanese people. However, when applying the FRAX ${ }^{\mathrm{TM}}$ model to Japan, Tscores and Z-scores should be converted into those derived from the international reference.

Conflicts of interest None. 


\section{References}

1. Orimo H, Sakata K (2006) The 4th nationwide survey for hip fracture in Japan (in Japanese). Japan Medical Journal 4180:25-30

2. Hagino H, Katagiri H, Okano T, Yamamoto K, Teshima R (2005) Increasing incidence of hip fracture in Tottori prefecture, Japan: trend from 1086 to 2001. Osteoporos Int 16:1961-1968

3. Kanis JA, Borgstrom F, De Laet C et al (2005) Assessment of fracture risk. Osteoporos Int 16:581-589

4. Kanis JA, Johnell O, De Laet C et al (2002) International variations in hip fracture probabilities: implications for risk assessment. J Bone Miner Res 17:1237-1244

5. Kanis JA, Black D, Cooper C et al (2002) A new approach to the development of assessment guidelines for osteoporosis. Osteoporos Int 13:527-536

6. Kanis JA on behalf of the World Health Organization Scientific Group (2008) Assessment of osteoporosis at the primary healthcare level. Technical Report. WHO Collaborating Centre, University of Sheffield, UK (in press)

7. Orimo H, Sugioka Y, Fukunaga M et al (1998) Diagnostic criteria of primary osteoporosis. J Bone Miner Metab 16:139-150

8. Orimo H, Hayashi Y, Fukunaga M et al (2001) Diagnostic criteria for primary osteoporosis: year 2000 revision. J Bone Miner Metab 19:331-337

9. Life Expectancy for Japanese (2003) Ministry of Health, Labor, and Welfare http://www.mhlw.go.jp/shinngi/2003/

10. Hagino H, Yamamoto K, Ohshiro $H$ et al (1999) Changing incidence of hip, distal radius, and proximal humerus fractures in Tottori prefecture, Japan. Bone 24:265-270

11. Fujiwara S, Kasagi F, Masunari N et al (2003) Fracture prediction from bone mineral density in Japanese men and women. J Bone Miner Res 18:1547-1553

12. Kanis JA, Oden A, Johnell O, Jonsson B, de Laet C, Dawson A (2001) The burden of osteoporotic fractures: a method for setting intervention thresholds. Osteoporos Int 12:417-427

13. Kanis JA, Oden A, Johnell O et al (2007) The use of clinical risk factors enhances the performances of BMD in the prediction of hip and osteoporotic fractures in men and women. Osteoporos Int 18:1033-1046

14. Kanis JA, Johnell O, Oden A, Johansson H, McCloskey EV (2008) FRAX ${ }^{\mathrm{TM}}$ and the assessment of fracture probability in men and women from the UK DOI 10.1007/s00198-007-0543-5
15. Looker AC, Wahner HW, Dunn WL, Calvo MS, Harris TB, Heyse SP (1998) Updated data on proximal femur bone mineral levels of US adults. Osteoporos Int 8:468-486

16. Johnell O, Kanis JA, Oden A et al (2005) Predictive value of bone mineral density for hip and other fractures. J Bone and Miner Res 20:1185-1194

17. De Laet C, Kanis JA, Oden A et al (2005) Body mass index as a predictor of fracture risk: a meta-analysis. Osteoporos Int 11:1330-1338

18. Ross PD, Fujiwara S, Huang C et al (1995) Vertebral fracture prevalence in women in Hiroshima compared to Caucasians or Japanese in the US. Int J Epidemiol 24:1171-1177

19. Oyama H, Fujiwara S, Masunari N, Hsu WL (2006) Comparisons between clinical vertebral fracture incidence and morphometric vertebral fracture incidence (in Japanese). Osteoporosis Jpn 148 (Suppl 1):147

20. Kanis JA, Borgstrom F, Zethraeus N, Johnell O, Oden A, Jonsson B (2005) Intervention thresholds for osteoporosis in the UK. Bone $36: 22-32$

21. Borgstrom F, Johnell O, Kanis JA, Jonsson B, Rehnberg C (2006) At what hip fracture risk is it cost-effective to treat? International intervention thresholds for the treatment of osteoporosis. Osteoporos Int 17:1459-1471

22. Eddy D, Johnston CC, Cummings SR et al (1998) Osteoporosis: review of the evidence for prevention, diagnosis and treatment and cost-effectiveness analysis. Osteoporos Int 8(Suppl 4): S7-S80

23. Kanis JA, Johnell O, Oden A et al (2005) Smoking and fracture risk: a meta-analysis. Osteoporos Int 16:222-228

24. Kanis JA, Johnell O, De Laet C et al (2004) A meta-analysis of previous fracture and subsequent fracture risk. Bone 35:375-382

25. Nakamura T, Turner CH, Yoshikawa $\mathrm{T}$ et al (1994) Do variations in hip geometry explain difference in hip fracture risk between Japanese and white Americans? J Bone Miner Res 7:1071-1076

26. Yan L, Crabtree NJ, Reeve J et al (2004) Does hip strength analysis explain the lower incidence of hip fracture in the people's republic of China? Bone 34:584-588

27. Iki M, Kagamimori S, Kagawa Y, Matsuzaki Y, Yoneshima H, Marumo F for JPOS Study (2001) Bone mineral density of the spine, hip, and distal forearm in representative samples of the Japanese female population: Japanese population-based osteoporosis (JPOS) study. Osteoporos Int 12:529-537 